\title{
The Kermanshah Acute Coronary Syndrome Registry: Rational and Design
}

\section{Hossein Siabani ${ }^{1}$, Patricia M Davidson ${ }^{2,3}$, Soraya Siabani ${ }^{1,3 *}$, Leila Gholizadeh ${ }^{3}$, Hossein Karim ${ }^{1}$, Farid Najafi ${ }^{1}$, Behrooz Hamzeh $^{1}$, Mohamad Reza Saeidi ${ }^{1}$, Maryam Babakhani ${ }^{1}$ and Atefeh Asadmobini ${ }^{1}$}

${ }^{1}$ Kermanshah University of Medical Sciences, Cardiovascular Research Centre, Iran

${ }^{2}$ The Johns Hopkins University, Baltimore, MD, USA

${ }^{3}$ University of Technology Sydney, Sydney, NSW, Australia

*Corresponding Author: Soraya Siabani, Kermanshah University of Medical Sciences, Cardiovascular Research Centre, Iran and University of Technology Sydney, Sydney, NSW, Australia.

Received: June 19, 2019; Published: July 15, 2019

DOI: $10.31080 /$ ASMS.2019.03.0354

\begin{abstract}
Background: The Kermanshah Acute Coronary Syndrome (KACS) Registry, funded by Kermanshah University of Medical Sciences (KUMS). Currently, the patients diagnosed by ST-elevation myocardial infarction (STEMI), are recruited. The registry is aimed to be expanded to include all patients with the diagnosis of ACS including STEMI, in 2022 subject to the success of current project.

Methods: In an ongoing cohort study, started July 2016, patients with a diagnosis of STEMI, being $\geq 18$ years old, are recruited. The study proposal has been approved by KUMS' Ethics committee and European Observational Registry Program (EORP). The baseline data include patients' characteristics, presenting symptoms, past medical history, clinical findings, medical procedures and paramedical tests are collected via patient interview and using a check list and questionnaire developed by EORP. The patients will be followed up annually for a minimum of two years.

Results: To date 2100 patients with STEMI have been recruited. Data on 220 variables for each participant has been collected. The first year follow up finished July 2018. We are analysing the results. An abstract entitled "Is any sex-specific difference in diabetes adverse effects on the outcomes of patients with myocardial infarction?" was presented at the 20th Asia Pacific Diabetes Conference in Australia (2018).The results gradually will be published.

Conclusions: The data in this registry will provide detailed epidemiologic characteristics and a list of risk factors, clinical features, therapeutic approaches provided for patients and their complications. It creates the possibility of investigation of such patients for next two years after their STEMI.
\end{abstract}

Keywords: Disease Registry; Surveillance; Myocardial Infarction; Cardiac Attack; Prevalence

\section{Abbreviations}

KACS: Kermanshah Acute Coronary Syndrome; KUMS: Kermanshah University of Medical Sciences; STEMI: ST-Elevation Myocardial Infarction; EORP: European Observational Registry Program; MI: Myocardial Infarction; ECG: Electrocardiogram; WHO: World Health Organization; CVD: Cardiovascular Disease; PCI: Percutaneous Coronary Intervention; HIS: Health Information System.

\section{Background}

Myocardial infarction (MI) refers to the presence of evidence of tissue necrosis associated with acute ischemic heart disease [1]. STsegment elevation myocardial infarction (STEMI) is the term used to describe a classic heart attack that is the most deadly sub-class of MI, and accounting for more than $35 \%$ of MI cases. The most common cause of myocardial infarction is coronary plaque rupture 
resulted from thrombus formation. If the plaque causes complete occlusion, acute STEMI will be the likely outcome [2]. The ST segment refers to the flat section of an electrocardiogram (ECG) representing the interval between jagged heartbeats that during a MI this section may elevate. MI can lead to major complications, such as electrical and mechanical disorders of the heart and ultimately the death of the patient. According to the World Health Organization (WHO) report in 2018, cardiovascular disease (CVD) is currently the most common cause of death among general population in both developed and developing countries [3]. In Iran, more than $40 \%$ of all deaths are caused by CVD [4].

The most common (80\%) cause of CVD deaths are due to MI and stroke [5,6]. Given the importance of MI in terms of incidence, prognosis, and its individual and economic burden, the WHO has considered this disease as the most important indicator of the prevalence of coronary artery disease in the community [1]. The incidence of MI was reported to be 73.3 per 100,000 people in 2012 in Iran [7]. In addition to the associated morbidity and mortality, CVDs imposes significant direct and indirect costs on the patient and health care system [8].

Timely diagnosis and effective management of MI through the use evidenced based approaches play a key role in improving the survival of the patients. Common treatment for MI includes the use of thrombolytic (fibrinolysis) agents or, preferably, primary percutaneous coronary intervention (PCI) that is the gold standard treatment for STEMI, if patient is treated in 12 hours of the event [2]. Yet, there are significant variations across health care institutions in management of MI and thus the disease outcomes [9,10]. Accordingly, the American Heart Association and the American College of Cardiology(2018) recommend measuring indicators of quality of care or performance measures to assess the effectiveness of interventions and disease management to improve outcomes for patients with CVD [11]. Therefore, it is necessary to carefully examine the treatment patterns and outcomes of patients with MI to ensure that the treatment approaches comply with the recommended guidelines $[9,10]$.

Although clinical trials provide valuable information on effectiveness of interventions, the characteristics of patients who participate in clinical trials may differ from the population, thus the results of clinical trials on patients with MI may not be generalizable to the wider population in a natural environment. This necessitates a systematic registration of patient information (clinical registry) [12].
A disease/patient registry is an organized system using observational study methods to collect clinical and personal data that are used to evaluate specific outcomes for a defined population suffering from a specific disease or living with a specific condition or being exposed to specific risk factors [13]. There are several registries for cardiovascular diseases in different countries. In the United States several registries related to CVD have been registered. In limited studies that have been carried out in Iran with the purpose of systematically recording and tracking MI data, data collection has mainly been based on patients' medical files and follow-up [1417]. Among such registries, there is a well-known cardiovascular disease registry in Isfahan conducted by Isfahan University of Medical Sciences which is running for seventeen years [18]. There are advance cardiovascular cohort-registries including 11 cardiovascular registries in Europe [19,20], a registry for acute myocardial infarction in Japan [21], KAMIRT registry in Korea [22], and the RENASICAII registry in Mexico [23], which can be used for modelling and benchmarking by other researchers and registries.

The objectives of the Kermanshah acute coronary syndrome (KACS) Registry

The KACS Registry aims to register information from all patients admitted to Imam Ali Hospital, Kermanshah with a diagnosis of STEMI and are equal or above 18 years old. The analysis of data collected through this registry will enable studying of epidemiologic status, risk factors, clinical features, therapeutic methods and outcomes of patients with STEMI in Western Iran. The specific objectives of the registry include:

1. To analyse data on demographic characteristics, medical history, and risk factors of patients with STEMI admitted to Imam Ali Hospital, Kermanshah, Iran.

2. To Present signs and symptoms of patients with STEMI in the study setting.

3. To access to pre- and-hospital care provided to the registered patients including the date and time of onset of symptoms, date and time of first medical contact, date and time of first contact with the doctor, type of first contact with the doctor, type of transfer to the hospital, date and time of the first ECG, date and time of arrival to Imam Ali Hospital, and care provided before arriving to the hospital.

4. Initial vital signs (including blood pressure, temperature and heart rate), initial ECG, Killip class, and ventilation time of patients with STEMI. 
5. The type of treatment method used including medical therapy, PCI and/or CABG.

6. The pathology tests while in hospital and the last pathology tests results,

7. including haemoglobin level, LDL cholesterol, creatinine, plasma glucose, total cholesterol, creatine kinase mb, troponin, platelet, white blood cells, uric acid.

8. Patients' status when leaving the hospital (e.g., critical condition, rehabilitation program, ECG rhythm, final diagnosis)

9. Complications of MI such as stroke, coagulated PCI, CABG, repeated infarction, stent thrombosis, mechanical complications, recurrent MI, tamponade, heart failure, atrial fibrillation during hospital stay.

10. Follow up data, including survival rate, recurrence of cardiac events, re-hospitalization, the experience of symptoms, and quality of life.

\section{Methods}

- Study design: This registry is a prospective cohort study, sponsored by Kermanshah University of Medical Sciences (KUMS).

- $\quad$ Setting: The registry is located in Imam Ali Hospital, a tertiary referral hospital in Kermanshah providence, West of Iran. The hospital offers the full spectrum of specialized cardiovascular services and diagnostics as well as cardiac rehabilitation.

- Participants: All patients with STEMI who attend or are referred to Imam Ali Hospital - the setting of study- are enrolled in the registry. The criteria for a patient to be included in the study are 1) a clinical history consistent with myocardial infarction; 2) electronic cardiograph (EKG) demonstrating STEMI; and being $\geq 18$ years old. Patients will be excluded if unwilling to participate in the study, or death before providing information. The eligibility of the patients to be enrolled in the study is assessed by an emergency medical doctor against the study criteria and consistent with the EROP protocol [24].

- Sample size: According to a pilot study conducted during a four months period in 2016, it is estimated that about 1000 patients with diagnosis of MI are admitted to Imam Ali Hospital annually. Considering the fact that about $80 \%$ of MI patients are diagnosed with STEMI, the enrolment will progress at the rate of 800 patients per year. Currently, the study has been funded by Kermanshah University of Medical Sciences to register patients for two years, allowing for enrolment of 1600 patients to the study over the next two years, with possible extension to 5 years subject to availability of fund. With the growing population and ageing society, the number of enrolled patients may be more than the estimated rate.

- Data collection and variables: The eligible patients, who sign the consent form to participate in the study, will be interviewed by one of the two trained nurses who will be present in the hospital on even days of the week, including public holidays. The interviews will begin after the patient admission to the hospital as soon as their health condition allows and data collection continues until the patient is discharged from the hospital. Data will include a wide range of demographics, risk factors, comorbidities, symptoms, diagnostic tests, therapeutic methods, patient's condition at discharge and follow up data as described above. The sources of data will include the patient or their relatives/care givers, and their medical records. A standard questionnaire adopted from the EORP will be used. The collected data will include information on different stages of MI trajectory including before admission to the hospital, during admission, during stay in the hospital and after discharge from hospital (a two year-follow up).

- Data quality control: A data dictionary has been created in order to explicitly define each question in the dataset and training provided to the interviewers. In addition, an expert medical doctor trained on the study protocol will review the completed questionnaires daily, before patients leave the hospital, and control the quality of collected data. Ultimately, the collected data will be sent to a quality control officer in the EORP for quality audit. A data base file has been developed for data entry, which will be later converted to SPSS format for data analysis.

\section{Data analysis}

After the quality of the collected data was confirmed by the quality control officer, data will be entered into the SPSS software and analysed using appropriate descriptive and inferential statistics, such as Chi-square, independent t-test, analysis of variance, or regression analysis.

- Ethical considerations: Participants will be provided with the participant information statement containing the purpose of the research and expectations. They will be ensured of the confidentiality of collected data and participant privacy will be maintained. Each participant will be assigned a code, which will be used in all forms and study questionnaires. All participants will sign a written consent before enrolling in the study. The study purpose and the related follow ups will also be explained to 
participants' next of kin, with permission from the patients, and their consent will be obtained for possible future contacts. Patients' information and collected data will be saved in password protected computers, placed in the Kermanshah Cardiovascular Centre in Imam Ali Hospital. Only three researchers and an EORP quality officer will be able to access the data.

- $\quad$ Follow up: First year and second year- follow up appointments will be arranged for all participants by the researchers. As part of the follow up arrangements, the participants will be examined by their doctor, and undergo various pathology and clinical tests, such as echocardiography. A researcher will then carry out a follow up interview with each participant to collect information on possible readmission to hospital due to CVD, including worsening of their symptoms, or occurrence of new cardiac event/s, such as a new MI or stroke, the related medical and interventional treatments and participants' subjective evaluation of their health.

\section{Limitations}

Some patients with acute MI, due to their health condition, may not be able to participate in the study interview. To address this limitation, a next of kin of the patient will be asked to provide the required information on the patient's behalf after obtaining the patient's consent. In cases where the patient's response is required, the information will be collected once the patient's health condition is stable, before discharge from the hospital.

Another likely limitation is 'loss to follow up'. To reduce loss to follow up, the participants will be asked to inform the researcher of their change of address or contact details. At least three contact numbers from the participant and their family members will be recorded during recruitment phase. Providing participants with information about the significance of the research as well as access to free of charge medical examinations, pathology tests, echocardiography and ECG, as part of the study should be appealing to the participants. Participants will be reimbursed for the cost of transportation if needed. Nevertheless, participation will be voluntarily and participants' decision to withdraw from the study will have no effect on their routine treatment. Follow up data will be collected through a home visit or a phone interview as much as possible, if a patient is not willing to attend the hospital for the follow up appointments. In addition, the hospital's health information system (HIS) will be used to track patient readmissions to the hospital over the study period. The death registry department of the Kermanshah state will be contacted if needed to confirm participant death. These strategies are aimed to reduce lost to follow up and enable collecting as much complete data as possible, according to the EORP protocol.

\section{Discussion and Conclusion}

Myocardial infarction imposes significant burden on individuals, society, and the health care systems worldwide. This study reports the protocol for forming a single site Iranian registry and surveillance system to obtain real-world information about current status of characteristics, risk factors, diagnosis, treatment and outcomes of patients admitted to Imam Ali Hospital with a diagnosis of STEMI. A clinical data registry can collect real patient information in real time and provide accurate health care information, which enable the comparisons of health care services and outcomes across health care institutions and against evidence-based guidelines. This provides the context for understanding the current health practices, existing strengths and weaknesses, resources needed as well as identifying priorities for action in order to improve outcomes for patients with STEMI. The health information that will be collected and stored through the KACS Registry will be invaluable to develop our understanding of the symptoms, pattern of access to care, before hospital care, diagnostic procedures, treatment modalities used, and disease and patient perceived outcomes in short and long terms.

The KACS Registry has received funding support from Kermanshah University of Medical Sciences to register the demographical and clinical/disease information of about 1600 patients with STEMI and follow up the patients for two years. The information gathered thorough this disease registry will enhance our knowledge about characteristics, disease symptoms, and management of patients with STEMI. This information are essential to evaluate the effectiveness of the current health care provision and therapies used and will base the foundation for development of future interventions to better improve the outcomes for patients with STEMI. The registry is aimed to be expanded to include all patients with diagnosis of acute coronary syndrome in future, subject to the success of current registry and availability of future funds.

\section{Declarations}

Ethics approval and consent to participate

Participants are provided with the participant information statement containing the purpose of the research and expectations. They will be ensured of the confidentiality of collected data 
and participant privacy will be maintained. All participants should sign a written consent before enrolling in the study. The Research Ethics Committee at Deputy of Research of the Kermanshah University of Medical Sciences (KUMS) has approved the study protocol in June 2016 (Ethics registration cod= KUMS.REC.1395.252).

\section{Consent for publication}

All authors of this manuscript wish the manuscript to be published by the journal of BMC Public Health. Also, they undertake the responsibility that this manuscript has not been published totally or partly (except abstract), accepted for publication or under peer- review for publication somewhere else.

\section{Availability of data materials}

Data are available for the corresponding author.

\section{Competing interests}

The authors declare that there were no conflicts of interest. In addition, the authors have no financial gain related to any aspect of the study.

\section{Funding}

This study was supported by Kermanshah University of Medical Sciences. The funding is for data collection process including quality control.

\section{Authors' contributions}

All authors have, fully or partly, been involved in the concepts and design of the study. In addition, all have reviewed the manuscript.

- Dr Hossein Siabania (cardiovascular researcher): Literature review, developing protocol, training data collectors, managing data collection and quality control, preparing manuscript

- Professor Patricia M Davidson (cardiovascular researcher): Developing proposal, scientific supervision of the process, reviewing manuscript critically

- Assistant Professor Soraya Siabani (cardiovascular researcher): Developing protocol, supervising data collection, analysing data, preparing manuscript and following up for publication

- Dr. Leila Gholizadeh (cardiovascular researcher): Literature review, consultation, reviewing manuscript critically

- Associate Professor Hossein Karim (Cardiologist): Literature review, following up for funding, scientific supervision of the process, reviewing manuscript critically
- $\quad$ Professor Farid Najafi (Epidemiologist): Epidemiological consultation, reviewing manuscript critically

- Associate Professor Behrooz Hamzeh (Health educatinist): Literature review, Developing the protocol, training and supervising data collection, reviewing manuscript critically

- Mohamad Reza Saeidia (Internal cardiologist): Preparing the required Facilities in research setting (hospital, cardiovascular consultation, reviewing manuscript critically.

- Ms Maryam Babakhania (BS of Public health): Literature review preparing the manuscript, reviewing manuscript critically

- $\quad$ Ms Atefeh Asadmobini (MS in cardiology): Literature review preparing manuscript, preparing the proposal, reviewing the manuscript critically

\section{Acknowledgment}

We would like to thank the Kermanshah University of Medical Sciences for funding this project and express our sincere appreciation for the high-quality collaboration of the Kermanshah Cardiovascular Research Center.

\section{Bibliography}

1. Thygesen K., et al. "Third universal definition of myocardial infarction". Journal of the American College of Cardiology 60.16 (2012): 1581-1598.

2. Uptodate. "Fibrinolysis for acute ST elevation myocardial infarction: Initiation of therapy" (2018).

3. WHO (2018).

4. Forouzanfar MH., et al. "Evaluating causes of death and morbidity in Iran, global burden of diseases, injuries, and risk factors study 2010". Archives of Iranian Medicine 17.5 (2014): 304-320.

5. Lozano R., et al. "Global and regional mortality from 235 causes of death for 20 age groups in 1990 and 2010: a systematic analysis for the Global Burden of Disease Study 2010". The Lancet 380.9859 (2013): 2095-2128.

6. Wong ND. "Epidemiological studies of CHD and the evolution of preventive cardiology". Nature Reviews Cardiology 11.5 (2014): 276-289.

7. Ahmadi A., et al. "Incidence of acute myocardial infarction in Islamic Republic of Iran: a study using national registry data in 2012". Eastern Mediterranean Health Journal 21.1 (2014): 5-12. 
8. World Health Organization. Cardiovascular diseases (CVDs), Fact sheet $\mathrm{N}^{\circ} 317$, Updated January 2015: World Health Organization (2015).

9. Fox KA., et al. "From guidelines to clinical practice: the impact of hospital and geographical characteristics on temporal trends in the management of acute coronary syndromes". European Heart Journal 24.15 (2003): 1414-1424.

10. Hasdai D., et al. "A prospective survey of the characteristics, treatments and outcomes of patients with acute coronary syndromes in Europe and the Mediterranean basin the Euro Heart Survey of Acute Coronary Syndromes (Euro Heart Survey ACS)". European Heart Journal 23.15 (2002): 1190-201.

11. Thomas RJ., et al. "2018 ACC/AHA Clinical Performance and Quality Measures for Cardiac Rehabilitation: A Report of the American College of Cardiology/American Heart Association Task Force on Performance Measures". Circulation: Cardiovascular Quality and Outcomes 11.4 (2018).

12. Zeymer U and Senges J. "Why do we need prospective registries in patients with acute myocardial infarction?" European Heart Journal 24.18 (2003): 1611-1612.

13. American Heart Association (2015).

14. Ahmadi A., et al. "Relationship between risk factors and inhospital mortality due to myocardial infarction by educational level: a national prospective study in Iran". International Journal for Equity in Health 13 (2014): 116.

15. Ghadimi H., et al. "Clinical characteristics, hospital morbidity and mortality, and up to 1-year follow-up events of acute myocardial infarction patients: the first report from Iran". Coronary Artery Disease 17.7 (2006): 585-591.

16. Najafi F., et al. "Long Term Survival and Hospital Admission Following Acute Coronary Syndrome a Cohort Study". International Journal of Health and Life Sciences 1.1 (2015): 7-16.

17. Sarrafzadegan N., et al. "Acute myocardial infarction in Isfahan, Iran: Hospitalization and 28th day case-fatality rate". ARYA Atheroscler 5.3 (2009).

18. The World Health Organization MONICA Project (monitoring trends and determinants in cardiovascular disease): a major international collaboration. WHO MONICA Project Principal Investigators. Journal of clinical epidemiology 41.2 (1988): 105-114.
19. Fox K., et al. "The ENACT study: a pan-European survey of acute coronary syndromes". European Heart Journal 21.17 (2000): 1440-1449.

20. EURObservational Research Programme Structure \& Organisation. Acute Coronary Syndrome STEMI (ACS) Registry EURO (2016).

21. Ishihara M., et al. "Clinical Presentation, Management and Outcome of Japanese Patients With Acute Myocardial Infarction in the Troponin Era-Japanese Registry of Acute Myocardial Infarction Diagnosed by Universal Definition (J-MINUET)". Circulation Journal 79.6 (2015): 1255-1262.

22. Kim HK., et al. "The scientific achievements of the decades in Korean Acute Myocardial Infarction Registry". The Korean journal of internal medicine 29.6 (2014): 703-712.

23. Al Suwaidi J. "RENASICA II: A Mexican acute myocardial infarction registry that highlights the importance of regional registries". Global cardiology science \& practice 2014.3 (2014): 232.

24. Roffi M., et al. "2015 ESC Guidelines for the management of acute coronary syndromes in patients presenting without persistent ST-segment elevationTask Force for the Management of Acute Coronary Syndromes in Patients Presenting without Persistent ST-Segment Elevation of the European Society of Cardiology (ESC)". European Heart Journal 37.3 (2016): 267315.

\section{Volume 3 Issue 8 August 2019 (C) All rights are reserved by Soraya Siabani., et al.}

